\title{
Preventive Interventions for Young Adults in Nightlife: Coproduction for a Systematic Literature Assessment Followed by a Stakeholder Dialogue Process
}

\author{
Judith Brunn Sibylle Brunner Margot Mütsch \\ Department of Epidemiology, Epidemiology, Biostatistics and Prevention Institute, University of Zurich, Zurich, \\ Switzerland
}

\section{Keywords}

Nightlife · Young people · Prevention · Stakeholder ·

Co-production · Substance use $\cdot$ Alcohol use

\begin{abstract}
Introduction: The nightlife-associated illicit drug and alcohol use presents diverse problems and includes different areas. In the Canton of Zurich, Switzerland, young adults in the nightlife setting were recently set as a priority group for preventive interventions. Method: Based on the predefined protocol, we systematically collated evidence on preventive interventions regarding young adults' use of alcohol and illicit drugs in nightlife. EBSCO Medline, Embase, Psyclnfo, and Psylndex were searched for reviews (1990-2016) and primary studies (2012-2016). Additional sources and experts were consulted, and stakeholders involved throughout the research process. Interventions were summarized according to the before-, at- and after-the-party stages. Results: Before the party, good-quality studies were found for social media interventions, indicating positive effects on alcohol consumption. For the at-the-party stage, good evidence of low to medium quality was presented for crisis interventions and medical care at festivals and for multi-sector approaches.
\end{abstract}

karger@karger.com www.karger.com/ear

Karger $\stackrel{\text { ' }}{5}$

GOPEN ACCESS
(C) 2021 The Author(s)

Published by S. Karger AG, Basel

This is an Open Access article licensed under the Creative Commons Attribution-NonCommercial-4.0 International License (CC BY-NC) (http://www.karger.com/Services/OpenAccessLicense), applicable to the online version of the article only. Usage and distribution for commercial purposes requires written permission.
The after-the-party setting was mainly covered by gray literature, and evidence remained limited for designated drivers and street safety interventions in the target group. The stakeholder dialogue was a structured exchange and favored the following evidence-informed preventive intervention fields: personalized feedback via social media, sustaining awareness among nightlife managers, focus on public nighttime transportation, and multi-sector approaches. Conclusion: The systematic involvement of stakeholders was an inspiring means for identifying evidence relevant for practice and policy in nightlife and fostering implementation. Especially, individual-based interventions, such as personalized feedback via social media and guided reflection on alcohol or drug use, and broader networking, were considered promising.

(c) 2021 The Author(s)

Published by S. Karger AG, Basel

\section{Introduction}

Since many decades, participating in nightlife has been one of the most favorite leisure and cultural activities of young Europeans [1]. Consistently, there is a strong association between nightlife participation and recreational 
alcohol and drug use [2,3]. According to the Global Drug Survey, the prevalence of recreational drug use among club patrons aged 15-34 years is about 4-25 times higher than in the general population of the same age [4]. Similar findings were reported for drunkenness, binge drinking, and pre-party drinking bearing risks for young adults, especially in nightlife [5-7].

Problems arising from the flourishing nightlife economy and the associated recreational substance use are diverse, context-sensitive, and include different features. They include social nuisance and health issues, such as dehydration or alcohol intoxication and long-term risks, often linked to psychiatric conditions (e.g., addiction and depression). Furthermore, substance-induced violence (including rape) and road safety linked to drunk driving are important concerns $[1,8]$.

On a national scale, $51 \%$ of Swiss bar patrons aged 1929 years reported use of psychoactive substances (excluding cannabis) on a typical "night out," with alcohol being most common. At the same time, $90 \%$ reported negative consequences of their substance use [9]. Furthermore, substance-induced violence is an important concern with $>70 \%$ of all assaults and physical injuries in Switzerland being alcohol-related [10]. Furthermore, alcohol-related fatal road crashes were monitored of having the highest incidence among people aged 18-24 years and during weekend nights [11].

For these reasons [9-11], young adults in the nightlife setting were recently set as a target group for future preventive interventions in the Canton of Zurich, Switzerland. Following that, relevant stakeholders asked for a targeted evidence synthesis and consented to bring in their perspective. Therefore, we embedded a systematic literature assessment coproduced with practice-based stakeholders and academia-based researchers in a structured stakeholder dialogue.

\section{Materials and Methods}

Based on the a priori protocol (in German), the research question covered preventive interventions regarding alcohol and illicit drug use among young adults in the nightlife setting.

\section{Data Sources and Search Strategy}

The following databases were searched for reviews and primary studies on August 29 and 30, 2016, respectively: EBSCO Medline, Embase, PsyIndex, and PsycInfo. Reviews and gray literature sources were included after 1990 and could be available in English, German, French, Spanish, or Italian. To access more recent data not yet included in reviews, primary studies were included from 2012 onwards. The search strategy was checked by an information specialist (see online suppl. File 1; for all online suppl. material, see www.karger.com/doi/10.1159/000511191). We also checked reference lists of included articles and asked Swiss experts about national and international nightlife interventions and unpublished reports.

Additionally, topic-based Web sites were searched: German Federal Center for Health Education (BzGA), German Center for the Control of Drug Abuse (DHS), Swiss National Center for Documentation of Public Health (CDSP), Addiction Switzerland, Swiss Research Institute for Public Health and Addiction, the European Monitoring Center for Drug and Drug Addiction (EMCDDA), Healthy Nightlife Toolbox, the Nightlife Empowerment \& Well-being Implementation Project (NEWIP), and the European Network for safer party labels (Party+). Furthermore, literature was searched in the Network of Libraries and Information Centers in Switzerland, Swissbib (catalog of Swiss university libraries), and the Karlsruhe Virtual Catalog and via Google.

\section{Selection of Reviews and Primary Studies}

Following de-duplication, 2 authors (J.B. and M.M.) independently performed the title and abstract screening and selected reviews and primary studies that met the inclusion criteria (outlined below). In case of discrepancies, consent was reached through discussion. References were managed using Mendeley (Elsevier), Covidence (www.covidence.org) for screening, and Microsoft Excel for the data extraction process.

\section{Types of Included Material}

We searched for all types of reviews, including systematic reviews and meta-analyses, scoping or rapid reviews, and qualitative or narrative syntheses. Primary studies could be interventional (e.g., randomized controlled trials (RCTs)) or observational studies (e.g., cross-sectional studies) and case reports. The gray literature included reports, working papers, institutional or governmental documents, academic theses, guidelines, conference proceedings, and book chapters.

\section{Type of Participants}

Young people aged $18-35$ years were included according to the NIH definition of "young adults" [12].

\section{Description of the Setting}

The "nightlife setting" was defined with the stakeholders as a social activity and entertainment taking place in public spaces (e.g., bars, clubs, parks, and festivals) after 8 p.m. We excluded interventions regarding private events, sport activities and bodybuilding, and sex worker and gay sex milieu.

\section{Types of Interventions}

Studies focused on preventive interventions targeting recreational drug and/or alcohol use in nightlife. According to the Dahlgren-Whitehead setting model [13], we considered approaches on the microlevel, such as individual- or group-centered approaches. They included brief interventions, personalized feedback, peer interventions, drug checking, and media campaigns. On the mesolevel, environmental approaches included club management practices (including house regulations, video monitoring, door policies, and design of the premises), staff trainings, public transport, night-lightings, and safety measurements in high-risk areas or care at festivals. Structural interventions were more on the macrolevel 


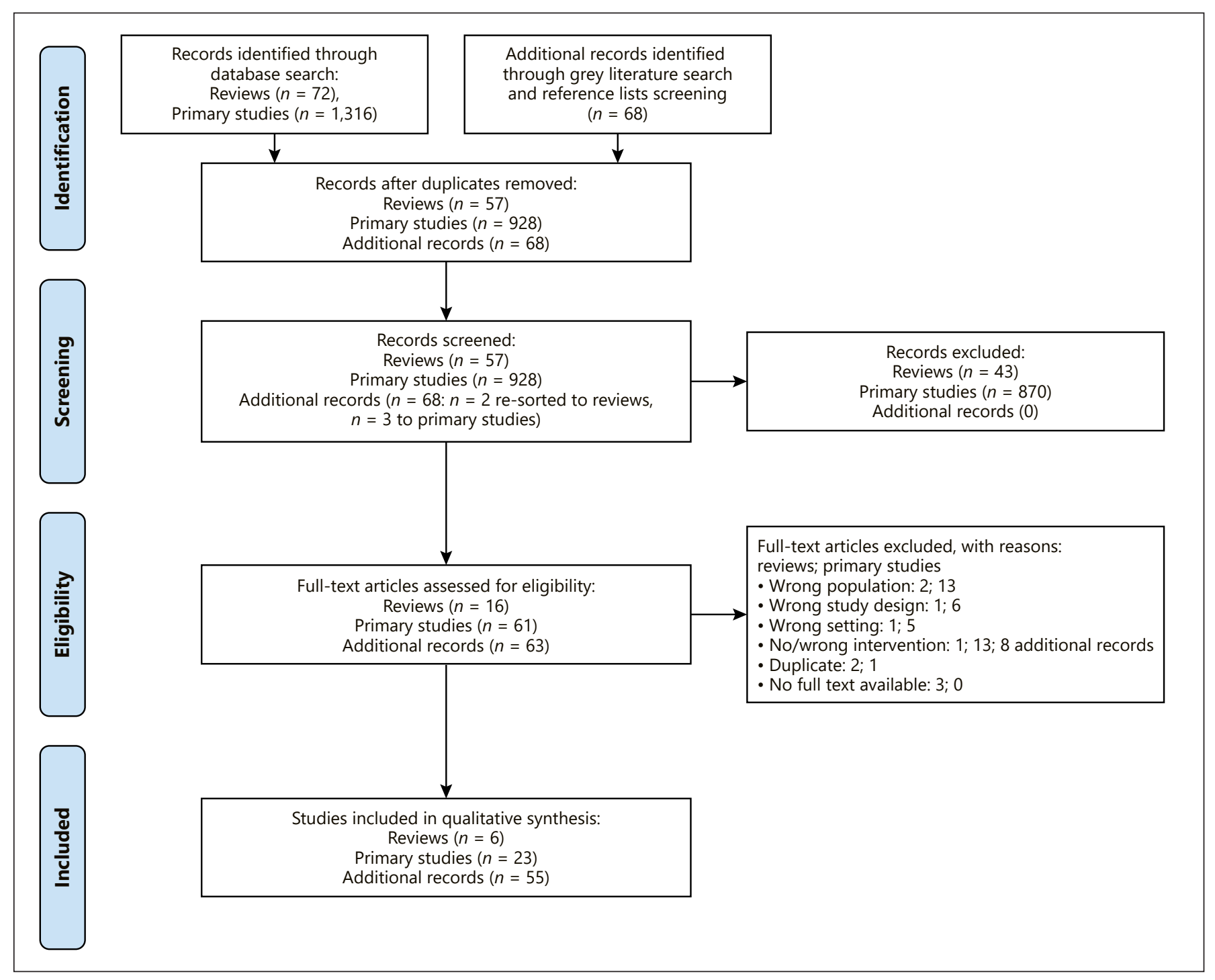

Fig. 1. PRISMA flow diagram of study selection.

and targeted inter-sectoral interventions, (inter-) national networking, knowledge exchange, and multi-sector cooperation involving, for example, police, party organizers, healthcare practitioners, party people, and policymakers. Legislative approaches and regulations regarding prices and package sizes as well as family- or school-based interventions were excluded, as they did not target the main scope of action of the stakeholders.

\section{Types of Outcomes}

Primary outcome dimensions of health literacy and harm reduction were assessed with "harm" referring to drunk driving and drug- or alcohol-related road accidents or violence, vandalism, nuisance, crime, injuries, mental crises, and presentations in emergency departments. Health literacy was defined according to the WHO as the ability to obtain, read, understand, and use health information in ways which promote and maintain good health. It is critical to empowerment and, therefore, includes motivation, behavioral intentions, and self-efficacy [14]. Secondary outcomes included alcohol or illicit drug consumption in nightlife (e.g., maximum or average number of drinks consumed per occasion, frequency of binge drinking, mean days of drug use during the previous weeks, and combinations of drug and alcohol) and cost-effectiveness.

\section{Data Extraction and Analysis}

Full-text articles were retrieved and assessed for inclusion independently by J.B. and M.M., with discrepancies resolved through discussion. Data were extracted using predefined standardized extraction forms. Of reviews and primary studies, we extracted country, year(s) of intervention, number and type of original studies, target group(s) of intervention, number of participants, target substance(s), setting, primary and secondary outcome results, and characteristics of the intervention. Outcomes included beneficial and harmful effects of the intervention(s). From the gray literature, 


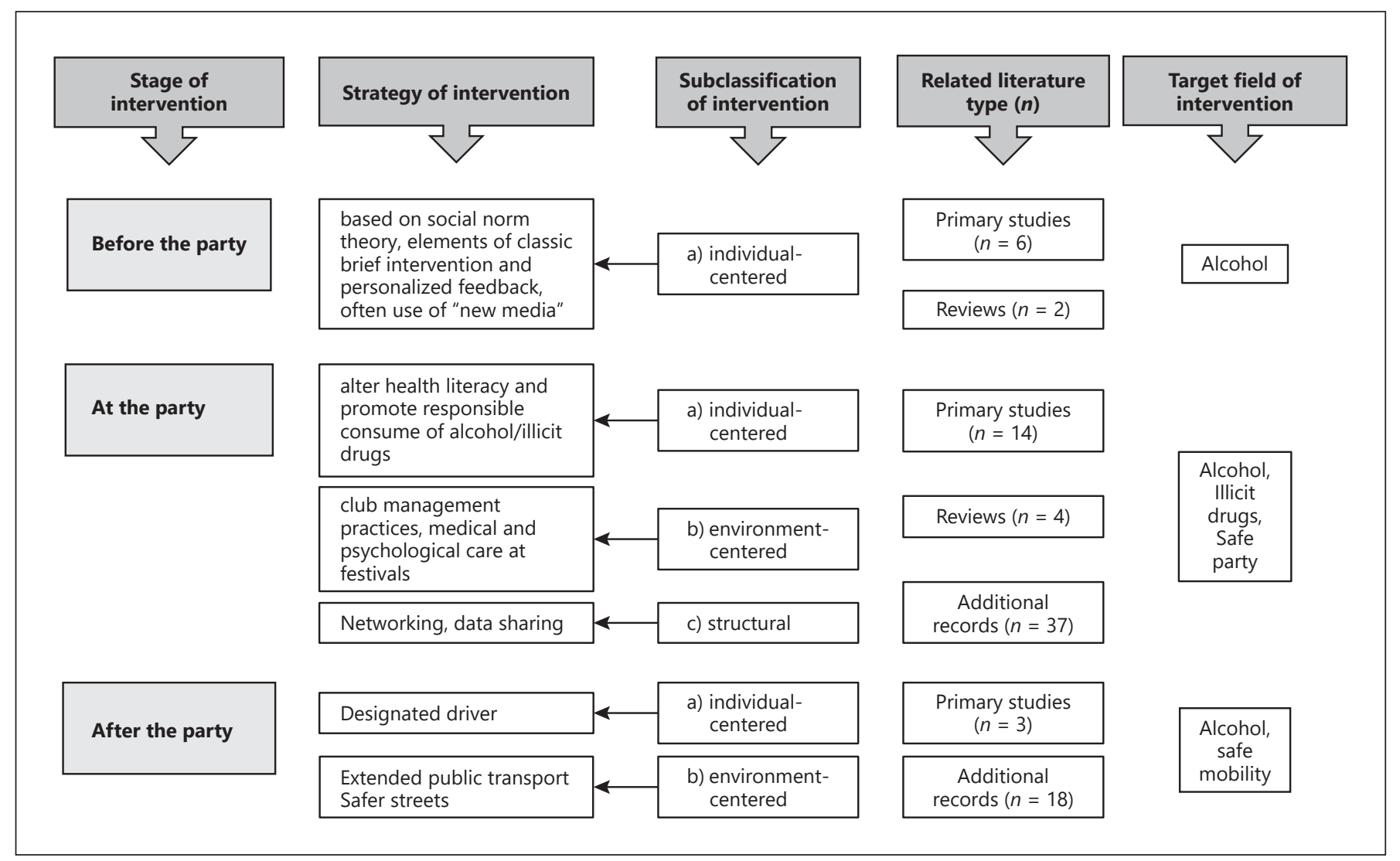

Fig. 2. Framework of the targeted nightlife setting.

we further extracted sponsorship, coordination body, and costand project evaluation-related data. Data extraction was performed by J.B. and spot-checked by M.M. on a random basis.

\section{Assessment of the Methodological and Reporting Quality of} Included Studies

The methodological quality of reviews was assessed using the Assessment of the Methodological Quality of Systematic Reviews (AMSTAR) tool, the reporting quality of the observational studies was assessed with the Strengthening the Reporting of Observational Studies in Epidemiology (STROBE) checklist. Furthermore, a 4-star rating system, established for systematic reviews and meta-analysis, was used [15]. Four stars were assigned to RCTs, 3 stars to uncontrolled experiments and controlled cohort studies, 2 stars to uncontrolled cohort studies, and 1 star to case reports. Due to missing instruments, the quality of the gray literature could not be assessed. All quality assessments were completed by J.B. and randomly spotchecked by M.M. Disagreements were resolved through discussion.

\section{Data Synthesis}

Data are presented as a narrative synthesis due to the heterogeneity of the study types. All evidence was grouped according to the starting point of the intervention as before the party, at the party, and after the party, and therein, references were assigned as individual-centered, environmental, or structural intervention.

\section{Knowledge Exchange with Stakeholder Involvement}

Knowledge exchange was defined as "a collaborative problemsolving between researchers and decision-makers through linkage and exchange involving mutual learning through the process of planning, producing, disseminating, and applying existing or new research in decision-making" [16]. As science and practice benefit from structured interaction, this case study in the nightlife field is of distinct relevance for an integrated knowledge exchange in the field of addiction. Methodologically, this process included the systematic literature assessment and its structured presentation as a deliberate dialogue $[17,18]$.

All invited stakeholders were mainly community-based experts in the field of addiction prevention, partly with an international focus. Most of them had a leadership role and long-standing practice in supervising the implementation of preventive interventions. In the systematic literature assessment, they were involved in scoping the research question and defining the interventions and outcomes to be targeted. The stakeholder dialogue was then designed to discuss the resulting evidence and its quality and to consider implementation options. It was structured by pre-formulated questions and according to the 3-party stages allowing participants to organize the presented input through building on their existing knowledge base and to interact, discuss, and further develop it according to the cognitive (learning) theory of Ausubel referred to by [19]. The starting point was to resolve questions, followed by the selection of especially relevant finding(s), the identification of similar interventions 
in Switzerland, the potential transferability to a Swiss context, and reasons for that. Here, we report the results of the discussion regarding especially relevant finding(s). The responses of the dialogue participants were recorded as a written protocol, which was submitted to the participants for additional feedback. The stakeholder dialogue was evaluated with a questionnaire adapted from Moat [20].

\section{Results}

Of the literature searched, 23 primary studies, 6 reviews, and 55 reports from gray literature and reference lists screening met the inclusion criteria. The primary studies included were not part of the reviews. The PRISMA flow diagram of the search process is depicted in Figure 1.

\section{Interventions with Respect to the Party Stage: before,} at, and after the Party

Most references targeted interventions at the party, including reviews $(n=4)$, primary studies $(n=14)$, and gray literature $(n=37)$. Literature published in this category was older (mostly before 2012) than for interventions before or after the party, respectively. Hence, interventions before the party were represented in more recent primary studies $(n=6)$ and reviews $(n=2)$, interventions focusing on the after-the-party stage were mostly represented by gray literature $(n=18)$ and some primary studies $(n=3)$, but reviews were missing. Most studies of good quality RCTs and reviews including RCT(s) were found for interventions at the before-the-party stage.

With respect to substance use, illicit drugs were less often targeted by primary studies and reviews $(n=1$ review) than alcohol ( $n=3$ primary studies). However, they were often at least co-targeted by peer interventions at parties, which often focused on "party safety" in general as shown in Figure 2.

All interventions starting before the party were individual-centered and referred to alcohol as target substance. Conceptually, they based on the social norm theory and included elements of brief intervention and personalized feedback. With 10 overlapping studies, there was considerable overlapping between the 2 reviews targeting brief interventions [21, 22]. They showed small positive short-term effects toward lower alcohol intake with a reduction of 1.5 drinks per week in heavy episodic drinkers and minus 0.8 drinking days per month among high school/university students, respectively [21, 22]. All European primary studies reported the use of social media apps, social networks, or serious games. They mostly showed positive effects on short-term drinking behavior
[23-27]. However, adverse effects were also reported, especially when including estimates of the blood alcohol concentration [28]. Thus, estimates of the blood alcohol concentration calculation lead to a significant increase in drinking frequency compared to controls in a Swedish study among university students with established levels of risky drinking [28]. Tables 1 and 2 depict the main characteristics of the included studies and the online suppl. Files 2-4 provide all data extracted.

Interventions focusing on the at-the-party stage were heterogeneous and included individual-centered approaches as well as environmental and structural perspectives, targeting alcohol as well as illicit drugs. Individual-centered approaches mostly included peer interventions, which aimed to foster health literacy and promote responsible consumption of alcohol or illicit drug use. According to an Australian primary study, these interventions were considered to lead to a better factual and theoretical knowledge on health hazards associated with the consumption of illicit drugs and on harm minimization strategies. There was also evidence of a decrease in illicit drug use 3 months following exposure to peer educators' information [29]. Moreover, computer-assisted interviews among young multidrug users decreased their drug use and substance dependence symptoms, especially after the first assessment, and were considered to support self-reflection [30]. On the other hand, better health knowledge did not necessarily lead to a consistent health behavior [31]. Of importance, primary studies reported the adverse impact of responsible drinking messages on drinking behavior under experimental conditions [32] or of blood alcohol level (BAC) tests in nightlife recreational settings $[33,34]$. Contrary to the intended effect, an even increased consumption of alcohol was favored. The gray literature mainly included professionals' attendance in nightlife to enhance health literacy and for harm reduction (including chill-out areas, provision of water, earplugs, and condoms) [9, 35-41]. Environmental approaches at the party included crisis interventions and medical care at festivals, which showed positive shortand long-term effects on the mental and physical health of users and lead to a decrease of emergency department admissions [42, 43].

On the other hand, contradictory data existed regarding the abundance and geographical distribution of alcohol-serving establishments and their association with drinking patterns [44, 45]. In Australia, voluntary licensing conditions on licensed venues did not reduce emergency department injury presentations during high-alcohol risk times compared to regulatory alcohol licensing 


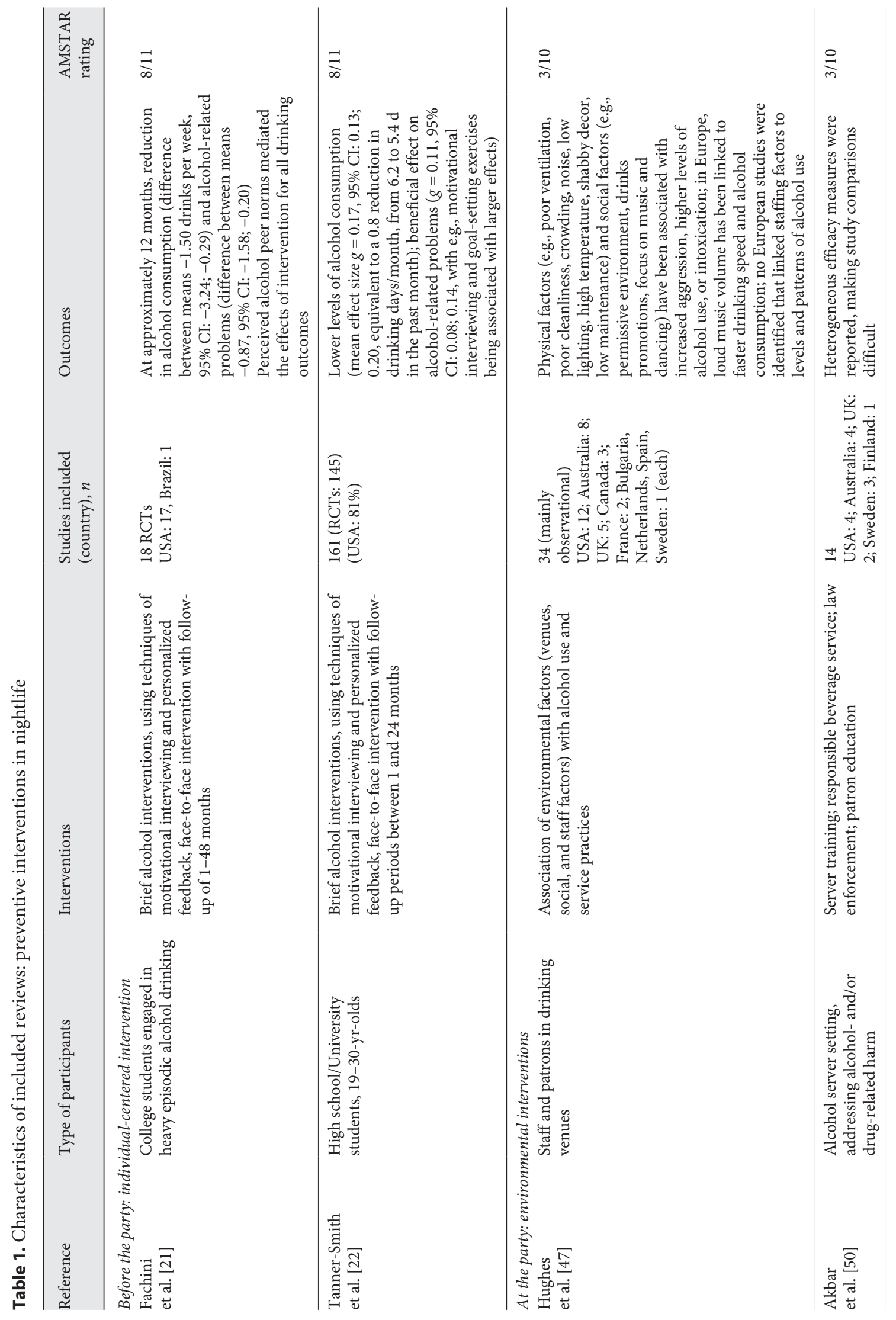




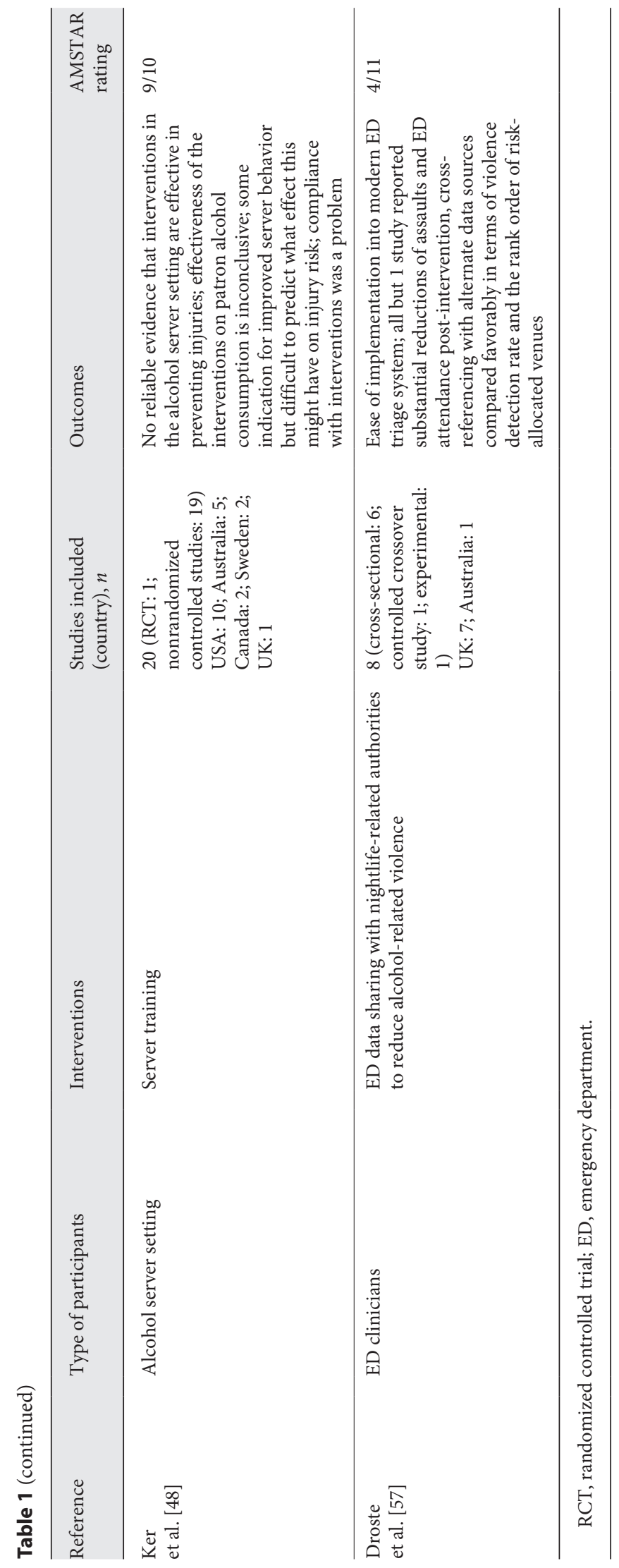

conditions [46]. Evidence regarding management practices in drinking venues (e.g., bar crowding, display of house and safety rules, door policy, and music volume) had been reviewed previously but remained inconclusive [47-49]. Two reviews were found to overlap with 2 studies $[48,50]$. Interventions described in the gray literature intended to improve the risk management at large events, such as providing "amnesty boxes" to deposit illicit drugs without prosecution and establishing safer clubbing networks [41, 51-55].

Structural approaches tended to optimize cooperation between educational strategies and enforcement, for example, regarding youth violence and appeared promising [56]. In a review of lower quality, emergency department data sharing to reduce alcohol-related violence via "hot spot" police interventions seemed to be effective in the UK [57]. One primary study described an online database to provide access to data of $>650$ novel psychoactive substances, a SMS information service, and multimedia information tools [58]. Interventions to provide trainings and guidelines $[59,60]$, as well as to support networking and media campaigning were addressed in the gray literature $[61,62]$. For more details, see online suppl. File 3.

Interventions paying attention to the after-the-party setting included individual-centered and environmental approaches to prevent drunk-driving and alcohol-related accidents and promote public transport [63-65]. In a 3 -year intervention period in Seattle, involving Spokane and Portland as comparisons, no overall effect of a multifaceted social marketing campaign was found among 21-34 year olds, but the use of designated drivers increased by $48 \%$ and the use of taxis by $63 \%$ among the heaviest drinkers. The crash data trends showed a decreasing trend in the last 18 months, whereas in the comparison cities and other age-groups no changes or increases were observed [63]. Especially, young people with a higher attitude to alcohol were more willing to use new transport options in Lugano, Switzerland, such as shared taxis. A combined strategy, including rising awareness of public transport alternatives and appropriate legislation, was found to increase this willingness [64]. The agentbased simulation model of Scott et al. [65] revealed a 25\% reduced prevalence of experiencing verbal aggression and an increase of alcohol consumption-related harms among heavy drinkers in Melbourne, Australia, with combined 24-h public transport operation and alcohol venue lockouts at 1 or 3 a.m. Furthermore, gray literature from the UK included safe travel advice by street patrols on weekend nights to increase street safety $[66,67]$ as summarized in online suppl. File 4. 


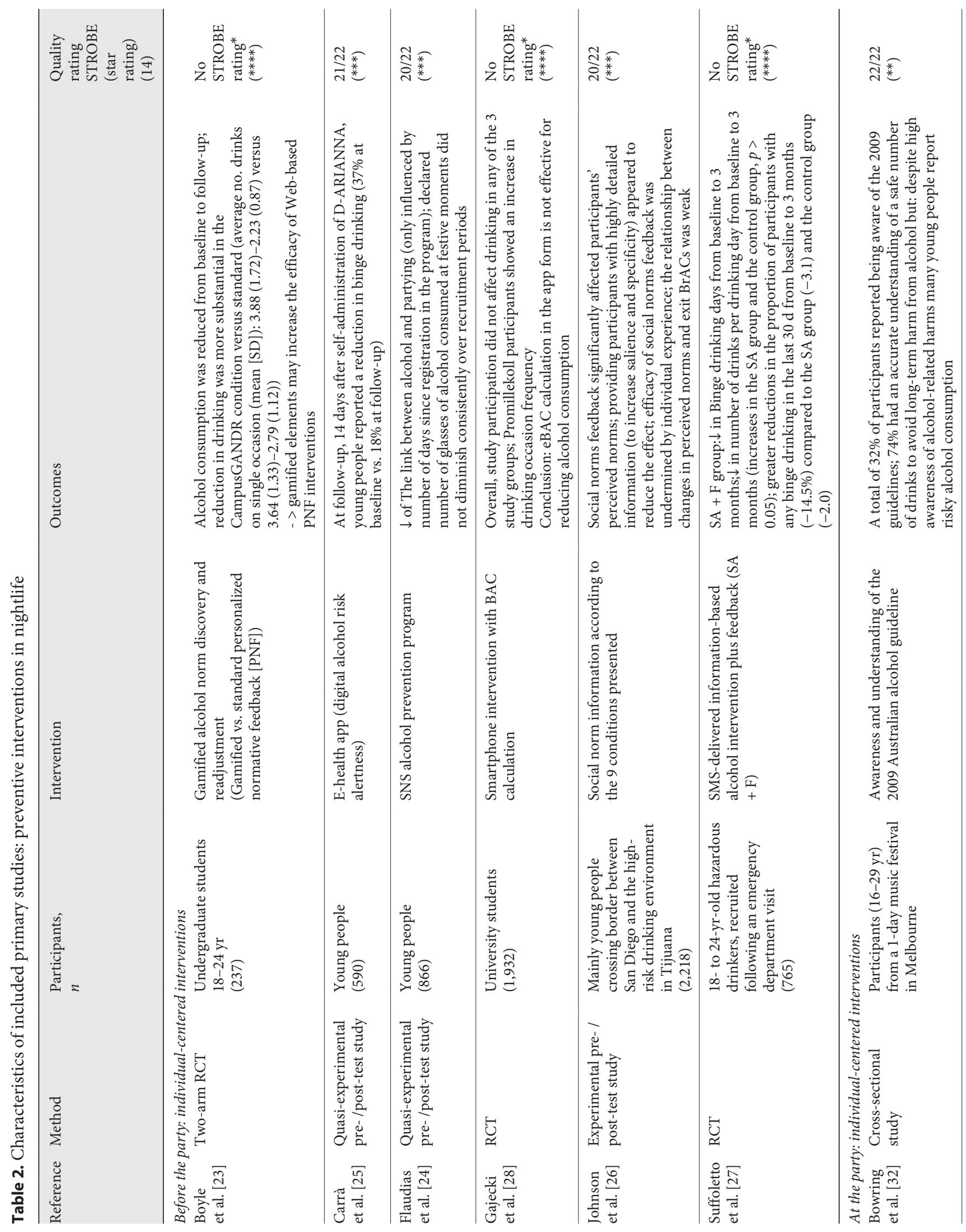




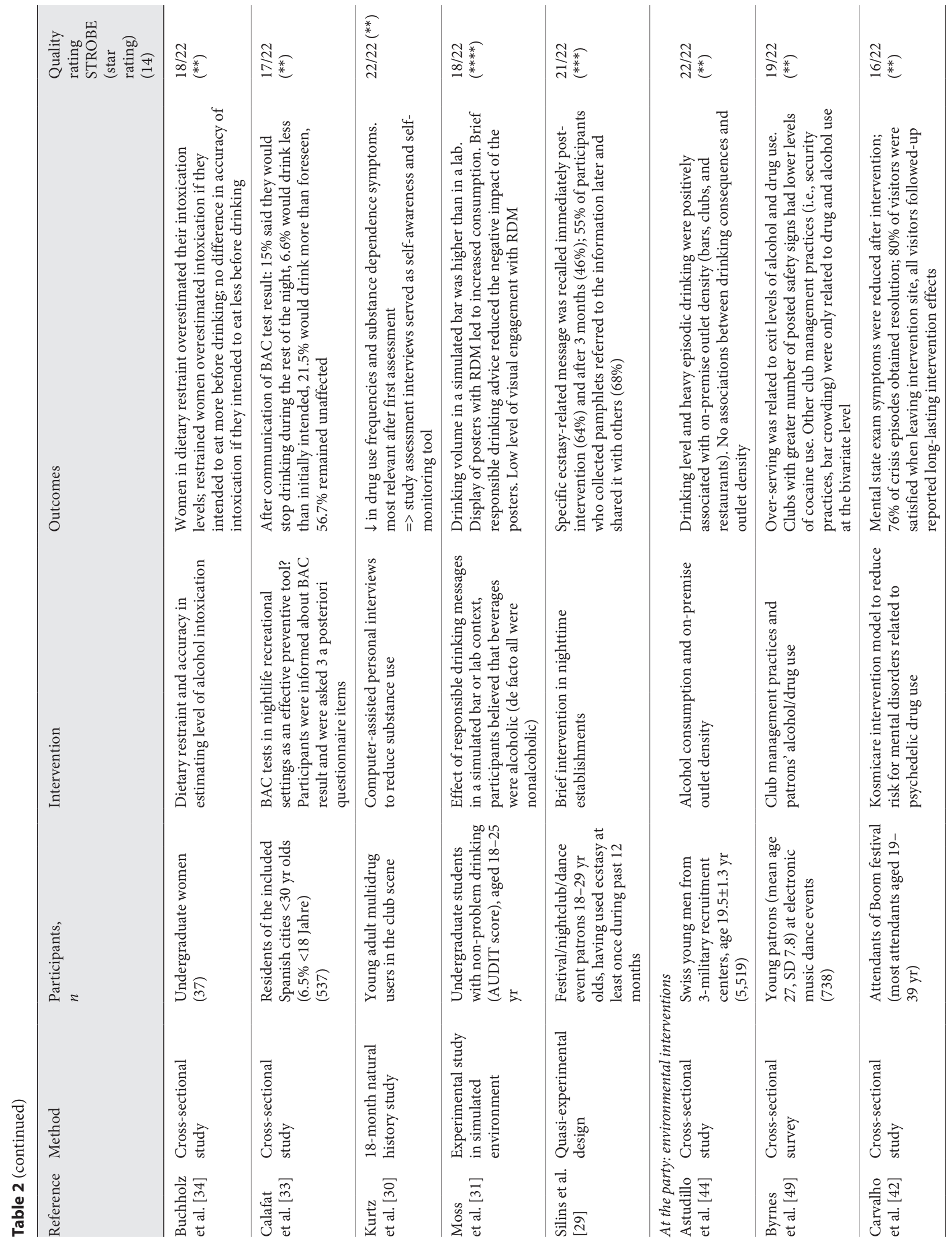




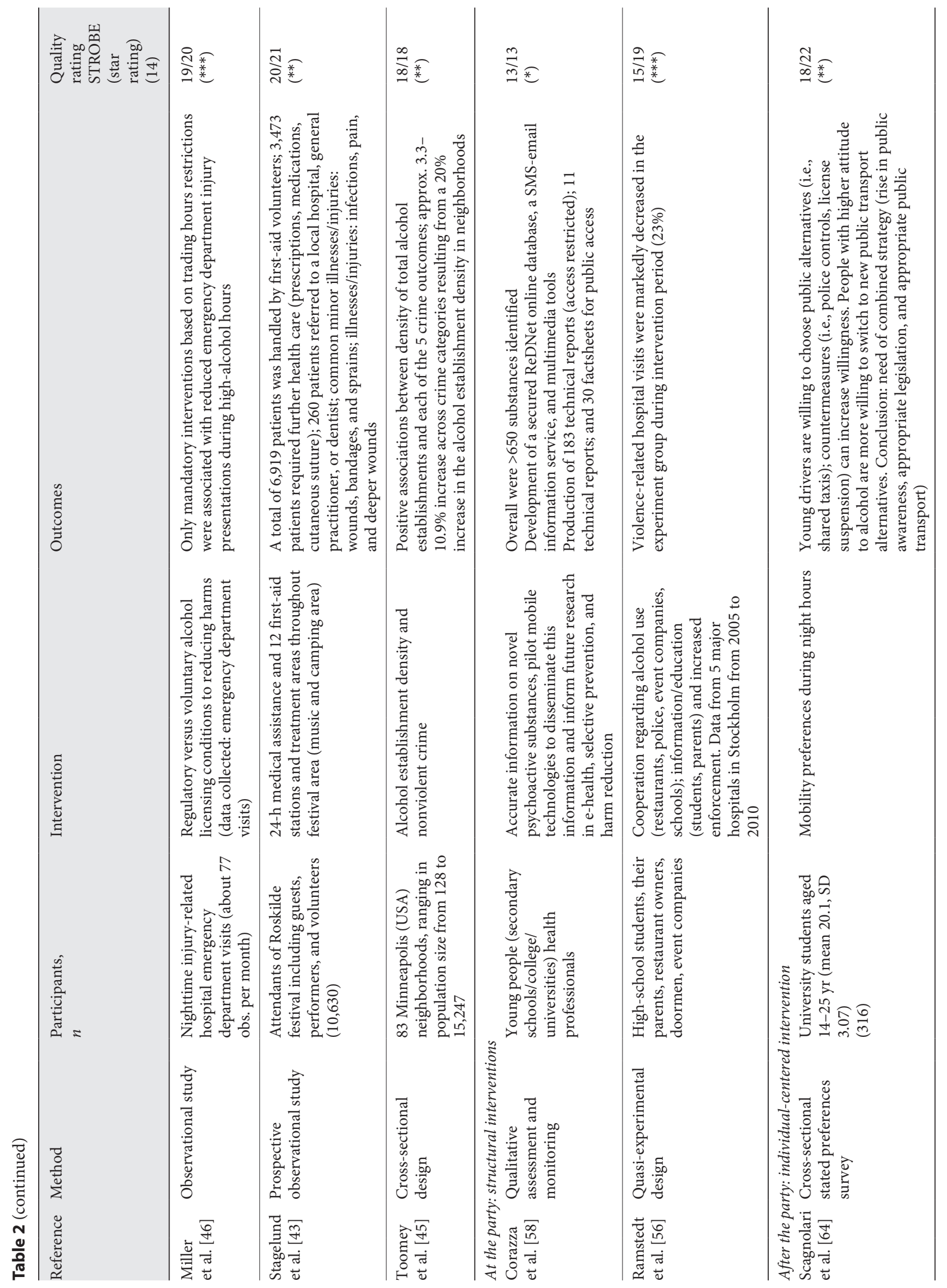




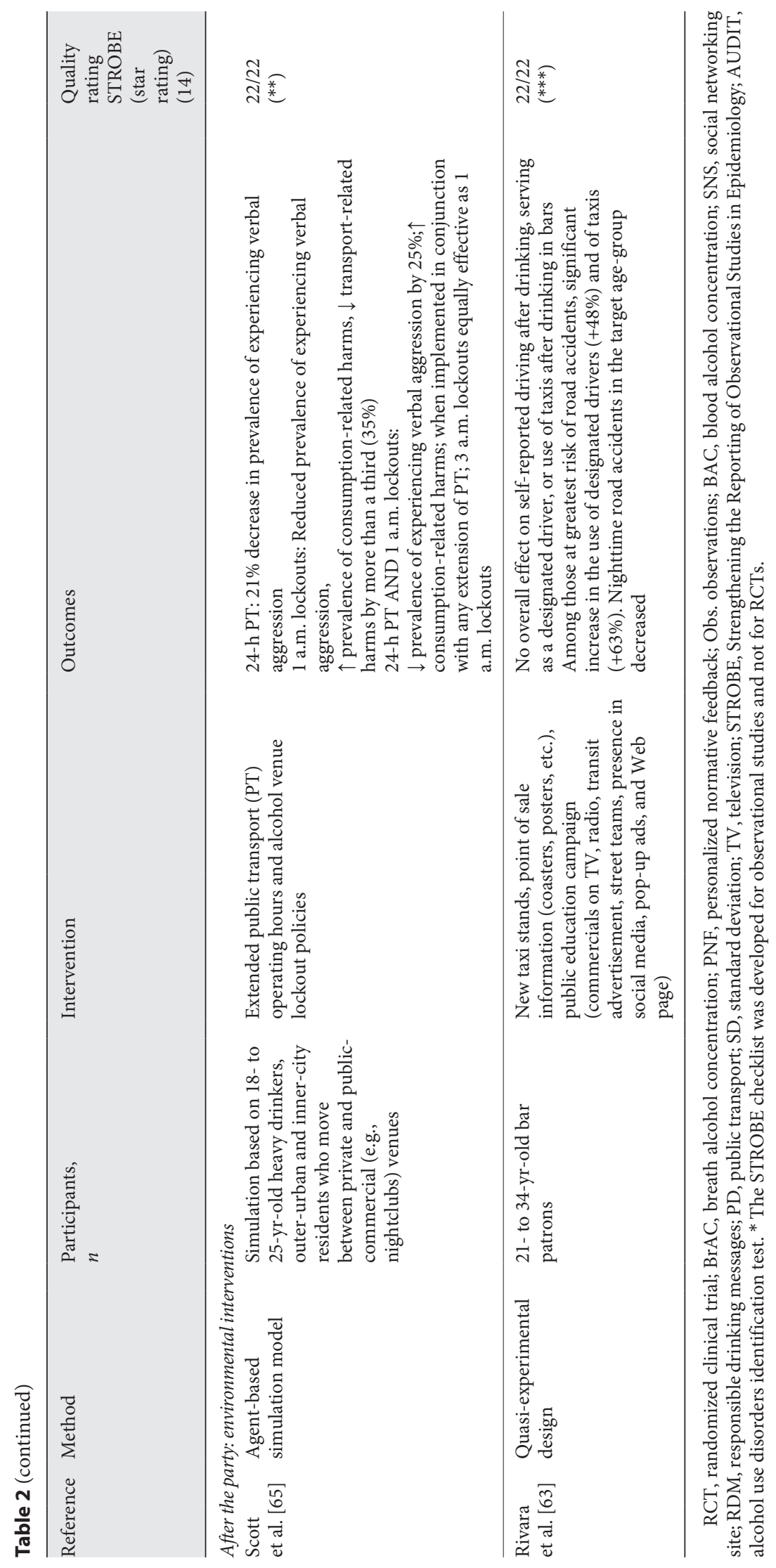




\section{Stakeholder Dialogue}

Following the evidence synthesis, a half-day stakeholder dialogue structured by the presentation of the findings and pre-formulated questions was implemented in July 2017. Prior to it, all 7 participants received a 2-page summary.

The following main aspects evolved during the discussion:

- self-reflection,

- health literacy,

- health behavior.

It was considered remarkable that interviews themselves influenced people's health behavior as described by Kurtz et al. [30]. This effect was mediated via interview questionnaires at baseline which made people reflect their own alcohol or drug consumption pattern and its negative consequences [30]. According to the stakeholders, this effect could also be seen in practice, for example, through interactions during drug checking. They were convinced that this could empower people to reconsider and improve their health knowledge and behavior.

On the other side, it was also shown that health literacy and health behavior were not always concordant. Thus, in an Australian study, many young festival patrons reported risky alcohol consumption, even though they had an accurate understanding of the safe number of drinks protecting them from long-term harm and injury [32]. Contrary to this, most of the Swiss young people were unaware of the nationally recommended drinking limits. Still, their alcohol consumption was considered less problematic than in most other countries, which was also reported in the Global Drug Survey and might be a cultural difference [68]. Hence, dialogue participants preferred health behavior-based outcomes (measured e.g., via alcohol or drug use) and drug-related harm measures (such as vandalism or emergency department admissions) for pragmatic reasons and because of its higher policy relevance.

Participants claimed that it was sometimes difficult to plan and evaluate preventive interventions targeting illicit drugs because of their illegal status that impaired open interactions. Thus, open discussions about drug problems and policies in nightlife establishments were difficult and stakeholder interests sometimes contradictory (e.g., regarding police and club management). However, participants recommended that optimally tailored interventions should always consider the whole nightlife context (private and public). This should envisage to prevent shifts of alcohol or drug-related harms from public to private sectors or to identify adverse events of preventive interventions [65].

\section{Personalized Feedback via Social Media}

The fact that BAC tests or their estimates could alter alcohol consumption and trigger an exploration of personal limits was consistent with the practical experience of the stakeholders. Additionally, the BAC was considered as a parameter of limited informational value. This was concordant with scientific evidence where mostly the level permitted to drive was used to inform people about the meaning of their BAC. Still, most people did not limit their consumption because of objective data but rather according to their personal feeling. In this context, "getting drunk" was rarely considered something adverse but often something to go for [33]. This was also the reason why stakeholders agreed that potential mobile apps should be customizable and focus on enjoyable consumption with minimized side effects, rather than on objective harm reduction. They should contain motivational, self-efficacy promoting contents, and support the critical reflection of the drinking behavior after the party, including individual cost-benefit analysis.

\section{Networking and Multi-Sector Approaches}

All participants agreed that knowledge exchange and networking facilitated a broader view and fostered mutual understanding. In future, especially a multi-sector approach [56] would merit further dissemination and was also supported by the European Monitoring Center for Drugs and Drug Addiction [69].

Participants agreed that emergency department data could present interesting outcome data for the planning of preventive interventions in a multi-sector approach. However, there were also constraints with respect to the setting and the patients being in a vulnerable situation (e.g., intoxicated and/or injured). Before local implementation, accordance with national data protection regulations and collaboration with hospitals and emergency department clinicians needed to be ensured. As an alternative, data of public nighttime transportation could be collected. Participants reported massive problems in this field (e.g., because of soiling and vandalism) and criticized that this area was underrepresented in multi-sector approaches. They further discussed to broaden the perspective and even integrate sporting events or holidays which were often confronted with similar challenges (e.g., binge drinking in holiday resorts or at sporting events).

\section{Evaluation of the Stakeholder Dialogue}

All participants provided written feedback. They agreed that it was helpful to consider scientific evidence 
when participating in decision-making processes. Still, for most of the participants, the use of evidence was not self-explanatory and they found it useful to discuss potential knowledge gaps, facilitators, and barriers regarding the implementation of evidence-informed practice and policy. Due to time constraints, more concrete strategies on how to proceed in future could only be developed at a subsequent meeting.

\section{Discussion}

We provided an overview of preventive interventions considering the nightlife spectrum, from the before- and at-the-party to the after-the-party stage. Therefore, the evidence synthesis and the stakeholder dialogue informed key stakeholders and researchers in an integrative way on exploring potential facilitators and barriers for evidence uptake and implementation in practice and policy. This was a major strength of our approach. The structured presentation of the findings, designed as an equal interaction between researches and practitioners allowed us to build relationships. We are convinced that not only the structured knowledge translation but also the relevant findings which emerged at the stakeholder dialogue might be of relevance for a European audience.

From a researcher's perspective, the heterogeneity of the included records was challenging when assessing their quality. The best available evidence, RCTs, mainly targeted individual-centered interventions in 3 articles $[23,27,28]$ and 2 higher quality reviews, which included mostly RCTs $[21,22]$. All of them addressed the beforethe-party stage. Another review included controlled studies and targeted with the alcohol server setting an environmental intervention at at-the-party stage. However, one should keep in mind that randomized controlled designs would not always be feasible, for example, for medical or psychological interventions at festivals.

No reviews of structural interventions of the after-theparty stage were found. This was also reflected by the high counts of gray literature in these fields which made it difficult to summarize evidence and impossible to perform critical appraisal of these interventions. Furthermore, dissemination into practice might be hindered by missing or unavailable evaluation measures.

At the stakeholder dialogue, participants mainly focused on individual-based and structural interventions. Regarding the former, personalized feedback via social media or apps and self-reflection measures, for example, performed as computer-assisted personal interviews, was considered as potential interventions for young adults in nightlife. Promisingly and described, for example, by a survey conducted in Berlin, Germany, partygoers themselves requested to combine the highly accepted drug checking with an educational offer about harms and risks of illicit drug use [70, 71].

They were preferred to responsible drinking messages (based on BAC limits or tests) and also to interventions striving health literacy-related outcomes, which were considered difficult to assess. However, and as a limitation of this study, the description of the term "health literacy" was not consistently used. Hence, selfreflection and personalized feedback enhancing self-efficacy would be part of health literacy if not a restricted definition is used based on provision of health information only.

The 2 reviews targeting brief alcohol interventions were mainly conducted in the US college or high school settings and their contextualization in a European setting might be questioned. Of note, there are brief alcohol intervention programs in Europe, targeting adolescents and young adults following alcohol-related emergency admissions. The respective review did not meet our inclusion criteria [72]. It described inconsistent evidence for effectiveness and feasibility and identified needs for further research [72], which might include the assessment of cultural factors.

Furthermore, stakeholders addressed networking and multi-sector approaches as structural interventions - although there is no high-quality scientific evidence available. They could include the before-the-party stage in addressing public and private nightlife as well as safety issues and public nighttime transportation after the party. Even an approach to target people's leisure time in general was envisaged including sport events with similar problematic alcohol or drug use, which could support the sharing of experiences. This would merit further research and a more integrated framework for the evaluation and contextualization could be provided by health promotion $[73,74]$ or the super-setting frameworks [75].

\section{Acknowledgements}

Our thanks go to Ms. Martina Gosteli and Mr. Diego Morosoli for performing the comprehensive literature searches. We thank all stakeholders for their engagement, their valuable and inspiring contributions, and discussions. 


\section{Statement of Ethics}

All authors confirm that this study complies with the guidelines for human studies. The research was conducted ethically in accordance with the World Medical Association Declaration of Helsinki.

\section{Conflict of Interest Statement}

The authors have no conflicts of interest to declare.

\section{Funding Sources}

The authors did not receive any funding.

\section{Author Contributions}

All authors designed the study and contributed to the protocol, and J.B. and M.M. performed the screening steps. Data extraction was done by J.B. with spot-checking by M.M., and all authors contributed and consented to the publication of the article.

\section{References}

1 Calafat A, Juan M, Duch MA. Intervenciones preventivas en contextos recreativos nocturnos: revisión. Adicciones. 2009;21(4):387414

2 Calafat A, Fernández C, Juan M, Becoña E. Recreational nightlife: risk and protective factors for drug misuse among young Europeans in recreational environments. Drug-Educ Prev Polic. 2008;15(2):189-200.

3 Fountain J, Griffiths P. Synthesis of qualitative research on drug use in the European Union: report on an EMCDDA project. European Monitoring Centre for Drugs and Drug Addiction. Eur Addict Res. 1999;5(1):4-20.

4 EMCDDA. European drug report 2015.

5 Gmel G, Rehm J, Kuntsche E. Binge drinking in Europe: definitions, epidemiology, and consequences. Sucht. 2003;49(2):105-16.

6 Ahlstrom S, Osterberg E. International perspectives on adolescent and young adult drinking. Alcohol Res Heal. 2004;28:258-68.

7 Sucht Schweiz. Erstes Schweizer Suchtpanorama 2015 Eine Politik der Widersprüche. 2015:1-21.

8 Bolier L, Voorham L, Monshouwer K, van Hasselt N, Bellis M. Alcohol and drug prevention in nightlife settings: a review of experimental studies. Subst Use Misuse. 2011; 46(13):1569-91.

9 Alexander M, Bachmann A, et al. Früherkennung und Frühintervention von problematischem Substanzkonsum im Schweizer Nachtleben: Synthesebericht der Ergebnisse der Datenerhebung 2011 bis 2013. Bern; 2014.

10 The Federal Council. National Strategy on addiction and action plan addiction: societal and individual responsibility. 2017.

11 Swiss Competence and Coordination Centre for Accident Prevention bfu. Assessing the effectiveness and efficiency of accident prevention measures in Switzerland, 2016-2017. 2020;1-3.

12 National Institutes of Health. Trials use technology to help young adults achieve healthy weights. [Internet]. Available from: https:// www.nih.gov/news-events/news-releases/trials-use-technology-help-young-adultsachieve-healthy-weights. Accessed $2016 \mathrm{Mar}$ 15. 2010.
13 Dahlgren G, Whitehead M. Policies and strategies to promote social equity in health. Background document to WHO: Strategy paper for Europe. Stockholm, Sweden.

14 Nutbeam D. Health literacy as a public health goal: a challenge for contemporary health education and communication strategies into the 21st century. Health Promot Int. 2000; 15(3):259-67.

15 Khan K, Kunz R, Kleijnen J, Antes G. Systematische Übersichtsarbeiten und Meta-Analysen. Ein Handbuch für Ärzte in Klinik und Praxis, sowie Experten im Gesundheitswesen. Berlin, Heidelberg, New York: Springer Verlag; 2004.

16 Graham ID, Logan J, Harrison MB, Straus SE, Tetroe J, Caswell W, et al. Lost in knowledge translation: time for a map? J Contin Educ Health Prof. 2006;26(1):13-24.

17 Lavis JN. Moving forward on both systematic reviews and deliberative processes. Healthc Policy. 2006;1(2):59-63.

18 Mulvale G, McRae SA, Milicic S. Teasing apart "the tangled web" of influence of policy dialogues: lessons from a case study of dialogues about healthcare reform options for Canada. Implement Sci. 2017;12(1):96-16.

19 Lawton JT, Saunders RA, Muhs P. Theories of piaget, bruner, and ausubel: explications and implications. J Genet Psychol. 1980;136(1): 121-36.

20 Moat KA, Lavis JN, Clancy SJ, El-Jardali F, Pantoja T. Evidence briefs and deliberative dialogues: perceptions and intentions to act on what was learnt. Bull World Health Organ. 2014;92(1):20-8

21 Fachini A, Aliane PP, Martinez EZ, Furtado EF. Efficacy of brief alcohol screening intervention for college students (BASICS): a meta-analysis of randomized controlled trials. Subst Abuse Treat Prev Policy. 2012;7:40-10.

22 Tanner-Smith EE, Lipsey MW. Brief alcohol interventions for adolescents and young adults: a systematic review and meta-analysis. J Subst Abuse Treat. 2015;51:1-18.

23 Boyle SC, Earle AM, LaBrie JW, Smith DJ. PNF 2.0? Initial evidence that gamification can increase the efficacy of brief, web-based personalized normative feedback alcohol interventions. Addict Behav. 2017;67:8-17.
24 Flaudias V, De Chazeron I, Zerhouni O, Boudesseul J, Begue L, Bouthier R, et al. Preventing alcohol abuse through social networking sites: a first assessment of a two-year ecological approach. J Med Internet Res. 2015; 17(12):e278-9.

25 Carrà G, Crocamo C, Bartoli F, Carretta D, Schivalocchi A, Bebbington PE, et al. Impact of a mobile e-health intervention on binge drinking in young people: the digital-alcohol risk alertness notifying network for adolescents and young adults project. J Adolesc Health. 2016;58(5):520-6.

26 Johnson MB. Experimental test of social norms theory in a real-world drinking environment. J Stud Alcohol Drugs. 2012;73(5): 851-9.

27 Suffoletto B, Kristan J, Callaway C, Kim KH, Chung T, Monti PM, et al. A text message alcohol intervention for young adult emergency department patients: a randomized clinical trial. Ann Emerg Med. 2014;64(6):66472.e4.

28 Gajecki M, Berman AH, Sinadinovic K, Rosendahl I, Andersson C. Mobile phone brief intervention applications for risky alcohol use among university students: a randomized controlled study. 2014;9(1):1-12.

29 Silins E, Bleeker AM. Does peer-delivered information at music events reduce ecstasy and methamphetamine use at three month follow-up? Findings from a quasi-experiment across three study sites. J Addict Prev. 2014; 2(1).

30 Kurtz SP, Surratt HL, Buttram ME, LeviMinzi MA, Chen M. Interview as intervention: the case of young adult multidrug users in the club scene. J Subst Abuse Treat. 2013; 44(3):301-8.

31 Moss AC, Albery IP, Dyer KR, Frings D, Humphreys K, Inkelaar T, et al. The effects of responsible drinking messages on attentional allocation and drinking behaviour. Addict Behav. 2015;44:94-101.

32 Bowring AL, Gold J, Dietze P, Gouillou M, Van Gemert C, Hellard ME. Know your limits: awareness of the 2009 Australian alcohol guidelines among young people. Drug Alcohol Rev. 2012;31(2):213-23. 
33 Calafat A, Fernández-Hermida JR, Becoña E, Juan M, Duch M, Fernández del Rio E, et al. Blood alcohol level tests in nightlife recreational settings as a preventive tool. Actas Esp Psiquiatr. 2013;41(1):10-6.

34 Buchholz LJ, Crowther JH, Olds RS, Smith KE, Ridolfi DR. Are restrained eaters accurate monitors of their intoxication? Results from a field experiment. Addict Behav. 2013;38(4):1966-9.

35 Arnaud S, Zobel F. Evaluation de la mise en oeuvre du projet "Nuit blanche" à Genève (2005-2006). Lausanne. 2006.

36 Carrasco S, Gervasoni JP, Dubois-Arber F. Projet-pilote sur la prévention et la réduction des risques en milieu festif nocturne. Lausanne. 2014.

37 Schaub MHIBA. Drug checking: a prevention measure for a heterogeneous group with high consumption frequency and polydrug use: evaluation of Zurich's drug checking services. Harm Reduct J. 2011;8:16.

38 Alexander M, Bachmann, Alwin LJB. Stimulanzienkonsum im Nachtleben. SuchtMagazin. 2013;39(3):15-20.

39 Maier LJ, Bücheli A, Bachmann A, Menzi P, Mikolasek M, Schaub MP. Stimulanzienkonsum im Nachtleben. Suchtmagazin. 2013;3: $15-20$.

40 Schaub MBAQ-HI. Evaluation der partydrogenprävention in der Stadt Zürich. SuchtMagazin. 2010;36(5):41-9.

41 Bücheli A, Menzi P. Reporting Safer Nightlife Schweiz (SNS) 2013. Bern; 2013.

42 Carvalho MC, De Sousa MP, Frango P, Dias $\mathrm{P}$, Carvalho J, Rodrigues M, et al. Crisis intervention related to the use of psychoactive substances in recreational settings: evaluating the Kosmicare project at boom festival. Curr Drug Abuse Rev. 2014;7(2):81-100.

43 Stagelund S, Jans $\varnothing$, Nielsen $K$, Jans $H$, Wildgaard K. Medical care and organisation at the 2012 roskilde music festival: a prospective observational study. Acta Anaesthesiol Scand. 2014;58(9):1086-92.

44 Astudillo M, Kuendig H, Centeno-Gil A, Wicki M, Gmel G. Regional abundance of onpremise outlets and drinking patterns among Swiss young men: district level analyses and geographic adjustments. Drug Alcohol Rev. 2014;33(5):526-33.

45 Toomey TL, Erickson DJ, Carlin BP, Quick HS, Harwood EM, Lenk KM, et al. Is the density of alcohol establishments related to nonviolent crime? J Stud Alcohol Drugs. 2012; 73(1):21-5

46 Miller P, Curtis A, Palmer D, Busija L, Tindall J, Droste N, et al. Changes in injury-related hospital emergency department presentations associated with the imposition of regulatory versus voluntary licensing conditions on licensed venues in two cities. Drug Alcohol Rev. 2014;33(3):314-22.
47 Hughes K, Quigg Z, Eckley L, Bellis M, Jones L, Calafat A, et al. Environmental factors in drinking venues and alcohol-related harm: the evidence base for European intervention. Addiction. 2011;106(Suppl 1):37-46.

48 Ker K, Chinnock P. Interventions in the alcohol server setting for preventing injuries. Cochrane Database Syst Rev. 2008(3):CD005244.

49 Byrnes HF, Miller BA, Johnson MB, Voas RB. Indicators of club management practices and biological measurements of patrons' drug and alcohol use. Subst Use Misuse. 2014;49(14): 1878-87.

50 Akbar T, Baldacchino A, Cecil J, Riglietta M, Sommer B, Humphris G. Poly-substance use and related harms: a systematic review of harm reduction strategies implemented in recreational settings. Neurosci Biobehav Rev. 2011;35(5):1186-202.

51 Webster Peter RJ. Drugs at the door: guidance for venues and staff on handling drugs. London. 2011.

52 Addiction valais. Rapport d' activité 2014 Secteur prévention. [in French]. 2014. Available from: http//cms.addiction-valais.ch/Upload/addiction-valais/News/Rapport\%20activite\%20prevention\%202014.pdf.

53 Schalbetter P, Mitarbeiterin W. Label Fiesta: Bericht über die 10 Jahre Tätigkeit und die Zufriedenheitsumfrage. 2014.

54 Safer Nightlife Projekte: Strategien zur Förderung von Safer Nightlife und effizienter Netzwerkarbeit: ein europäischer Ansatz. 2007.

55 Safer nightlife arbeitskreis 2005-2007 (leitung: energy control). Safer Nightlife Projects, 2007.

56 Ramstedt M, Leifman H, Müller D, Sundin E, Norström T. Reducing youth violence related to student parties: findings from a community intervention project in Stockholm. Drug Alcohol Rev. 2013;32(6):561-5.

57 Droste N, Miller P, Baker T. Review article: emergency department data sharing to reduce alcohol-related violence: a systematic review of the feasibility and effectiveness of community-level interventions. Emerg Med Australas. 2014;26(4):326-35

58 Corazza O, Assi S, Simonato P, Corkery J, Bersani FS, Demetrovics Z, et al. Promoting innovation and excellence to face the rapid diffusion of novel Psychoactive substances in the EU: the outcomes of the reDNet project. Hum Psychopharmacol. 2013;28(4):317-23.

59 Quigg Z, Ford K, Butler N, Hardcastle K, Hughes K. Evaluation of the South Wales Know the score intervention. 2015.

60 Grey H, Quigg Z. Evaluation of the Wirral's drink less enjoy more intervention. 2016.

61 Leclercq D, Noijen J, Charlois T, Allart M, Akeret R, Galan V, et al. Safer nightlife: labels and charters: good practice standards. 2012

62 Widmer T, De Rocchi T, Stutz M. Externe Situationsanalyse: Netzwerk Safer Nightlife Schweiz. Bern Bundesamt für Gesundheit. $2015 ; 72$.
63 Rivara FP, Boisvert D, Relyea-Chew A, Gomez T. Last call: decreasing drunk driving among 21-34-year-old bar patrons. Int J Inj Contr Saf Promot. 2012;19(1):53-61.

64 Scagnolari S, Walker J, Maggi R. Young drivers' night-time mobility preferences and attitude toward alcohol consumption: a hybrid choice model. Accid Anal Prev. 2015;83:7489.

65 Scott N, Hart A, Wilson J, Livingston M, Moore D, Dietze P. The effects of extended public transport operating hours and venue lockout policies on drinking-related harms in Melbourne, Australia: results from SimDrink, an agent-based simulation model. Int J Drug Policy. 2016;32:44-9.

66 Tackling alcohol problems in the night time economy: guidance for local areas. London. 2012.

67 Bücheli A. Schadensminimierung im Schweizer Nachtleben. SuchtMagazin. 2014; 40(2):16-23.

68 Winstock A, Barret M, Ferris J, Maier L; The GDS Core Research Team. What we learned from GDS2016 an overview of our key findings. [Internet]. Available from: https://www. globaldrugsurvey.com/wp-content/uploads/2016/06/TASTER-KEY-FINDINGSFROM-GDS2016.pdf. 2016.

69 European Monitoring Centre for Drugs and Drug Addiction. Responding to drug use and related problems in recreational settings. 2012:1-12.

70 Betzler F, Ernst F, Helbig J, Viohl L, Roediger L, Meister S, et al. Substance use and prevention programs in Berlin's party scene: results of the SuPrA-study. Eur Addict Res. 2019; 25(6):283-92.

71 Betzler F, Helbig J, Viohl L, Ernst F, Roediger L, Gutwinski S, et al. Drug checking and its potential impact on substance use. Eur Addict Res. 2020:1-8.

72 Diestelkamp S, Drechsel M, Baldus C, Wartberg L, Arnaud N, Thomasius R. Brief in person interventions for adolescents and young adults following alcohol-related events in emergency care: a systematic review and European evidence synthesis. Eur Addict Res. 2016;22(1):17-35.

73 Liveng A, Andersen HM, Lehn-Christiansen S. Health promotion in context: a reflectiveanalytical model. Scand J Public Health. 2018; 46(20 Suppl):66-71.

74 Best A, Stokols D, Green LW, Leischow S, Holmes B, Buchholz K. An integrative framework for community partnering to translate theory into effective health promotion strategy. Am J Health Promot. 2003;18(2):168-76.

75 Bloch P, Toft U, Reinbach HC, Clausen LT, Mikkelsen BE, Poulsen K, et al. Revitalizing the setting approach: supersettings for sustainable impact in community health promotion. Int J Behav Nutr Phys Act. 2014;11(1): $118-5$. 